\title{
SIMULATION ALS INTERPRETATIONSINSTRUMENT FÜR ERGEBNISSE STATISTISCHER ANALYSEN
}

\author{
Jochen Benz, Fachhochschule Konstanz
}

Die Interpretation von Ergebnissen statistischer Erhebungen sowie deren Umsetzung in konkrete Maßnahmen bereiten in der Praxis oft erhebliche Schwierigkeiten. Dies ist insbesondere dann zu beobachten, wenn Multivariate Methoden für die Datenanalyse zum Einsatz kommen.

Eine Hilfestellung können beispielsweise Wissensbasierte Systeme liefern. Insbesondere für die Interpretation und Demonstration von Ergebnissen linearer Strukturmodelle bietet sich jedoch die Simulation als Instrumentarium an. Hierbei ist es unerheblich, ob diese Kausalanalysen mittels einer Kombination "traditioneller Verfahren" oder simultaner Methoden durchgeführt wurden.

Hybride Expertensystem-Shells - wie zum Beispiel Kappa-PC - erlauben sowohl die Gestaltung von Wissensbasierten Systemen als auch von Simulationsmodellen mit relativ geringem Programmieraufwand.

In diesem Beitrag soll die Realisierung eines Simulationsmodells zur Interpretation von Kausalanalyseergebnissen vorgestellt werden. Zielsetzung dieses Modells ist es, die Erarbeitung konkreter Maßnahmen zur Verbesserung von Schwachstellen, aufgedeckt mittels statistischer Daten und Analysen, zu unterstützen.

Der Anwender hat die Möglichkeit, an das System "was-wäre-wenn-Fragen" zu stellen. Damit erhält er einen Eindruck davon, wie sich einzelne Maßnahmen auswirken. Zugleich hat er aber auch die Möglichkeit, komplette Szenarien in kurzer Zeit durchzuspielen.

Da das vorgestellte System mittels einer hybriden Expertensystem-Shell entwickelt wurde, demonstriert es gleichzeitig die Mächtigkeit dieser Werkzeuge. 